\title{
SUPERSEISMIC LOADING AND SHOCK POLARS: AN EXAMPLE OF FLUID-SOLID COUPLING
}

\author{
Marco Arienti and Joseph E. Shepherd
}

\author{
Graduate Aeronautical Laboratories, California Institute of Technology, Pasadena, CA 91125
}

\begin{abstract}
We propose a two-dimensional problem involving fluid-solid coupling where a solution is given in closed form. The upper half of the domain is modeled as an isotropic solid; the lower part as a compressible gas. The loading of the solid at the fluid-solid boundary is called superseismic when its speed is larger than the speed of propagation of disturbances in the bulk of the material. The loading is modeled by a shock coupled to the deformation of the boundary. The problem is relevant to high explosive applications, since it is very similar to the interaction between an explosive and the casing in a cylinder test experiment. Within this framework, we show the existence of self-similar solutions in the reference frame moving with the shock wave.
\end{abstract}

\section{INTRODUCTION}

We consider wave interactions between a solid and a compressible fluid. The twodimensional domain is divided into two parts, see Fig. 1. The contact discontinuity at the division is labeled the fluid-solid interface.

A compressible fluid occupies the bottom half of the domain (regions 1, 2, 3 and 4). Its flow is described by the inviscid Euler equations. Here, the perfect gas Equation of State (EoS) is used for simplicity.

A deformable solid occupies the upper half of the domain (regions $0,5,6$ and 7). A closed form solution describing the effect of a step-load over a half plane is provided by Bleich [1] when the boundary load travels faster than any bulk disturbances in the solid (superseismic loading).

We will consider the system of waves generated by a shock advancing in a fluid at rest (region 1 in Fig. 1) and at an angle with the interface. When the deformation produced by the shock load on the solid does not generate an ap- preciable feedback in the fluid, the interface can be treated as rigid. This case has been extensively studied, see for instance [2].

Conversely, the solid material can be treated as a fluid by neglecting the deviatoric components of the stress tensor. This approximation is typically adopted in high explosives applications [3] where the explosive confinement undergoes extremely high compressions.

Here, we propose a model for a fluid-solid interaction that is somewhat intermediate between the two indicated above. The interface deformation has a significant effect on the fluid, but the solid confinement retains its shear strength. As in the previous cases, self-similar solutions exist in a shock-attached reference frame.

\section{SUPERSEISMIC LOADING}

In this paper, superseismic loading is applied in the case of plane strain $\left(\varepsilon_{\dot{z}}=0\right)$ in an isotropic homogeneous material. The load is applied in the undeformed configuration to be consistent with the hypothesis of small strains. 


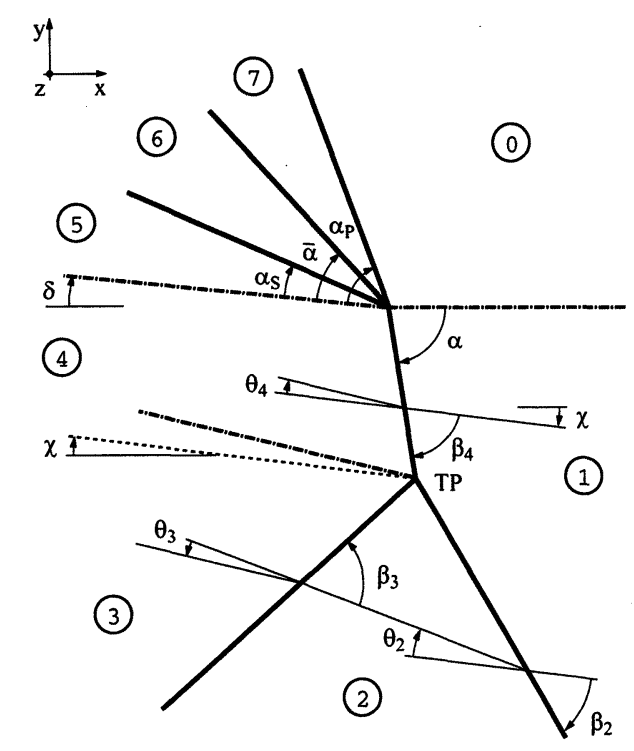

FIGURE 1. Mach Reflection for fluid-solid interaction.

Stress waves propagate into a semi-infinite medium that supports shear at two different speeds corresponding to the propagation of dilatational and distortional disturbances

$$
\begin{gathered}
c_{P}=\sqrt{(\lambda+2 \mu) / \rho}, \\
c_{S}=\sqrt{\mu / \rho} .
\end{gathered}
$$

The parameters $\lambda$ and $\mu$ are the Lamé constants, and $\rho$ is the density of the medium. We will refer to these waves as dilatational $(p)$ and distortional $(s)$ waves, respectively.

When a boundary load $P$ travels with a speed $D$ larger than $c_{P}$, two plane waves are produced. They form characteristic angles with the boundary of the half space

$$
\begin{aligned}
& \tan \alpha_{P}=\sqrt{D^{2} / c_{P}^{2}-1}, \\
& \tan \alpha_{S}=\sqrt{D^{2} / c_{S}^{2}-1} .
\end{aligned}
$$

If region 0 is undeformed, the wedge area between the $p$ and $s$ waves is in a state of uniaxial strain, whereas the distortional wave introduces a discontinuity in shear. In the linear elastic case, there is no discontinuity between regions 6 and 7 and the separation at angle $\alpha$ is immaterial. Following Bleich, the stress tensor is a function of the parameter $N$

$N=\frac{1}{2}\left(\cos 2 \alpha_{S}+(1-2 \nu) \cos 2\left(\alpha_{S}-\alpha_{P}\right)\right)$

where $\nu$ is the Poisson ratio of the material.

In linearized elasticity we can sum the effects due to the $s$ and $p$ fronts. The expression for the Lagrangian velocities at the interface $(x-y$ reference) is derived in [4]

$$
\begin{aligned}
& u=\frac{D(1-2 \nu) P}{4 \mu N}\left(\cos 2 \alpha_{S}-\cos 2\left(\alpha_{P}-\alpha_{S}\right)\right), \\
& v=\frac{D(1-2 \nu) P}{4 \mu N}\left(\sin 2 \alpha_{P}\right) .
\end{aligned}
$$

When the confinement undergoes plastic deformation, the above relations are no longer valid. To describe the behavior of an elasticplastic material the yield function is introduced

$$
F=J_{2}^{\prime}-k^{2} \quad F \leq 0
$$

where $J_{2}^{\prime}$ is the second stress invariant

$$
J_{2}^{\prime}=\frac{\left(\sigma_{1}-\sigma_{2}\right)^{2}+\left(\sigma_{2}-\sigma_{3}\right)^{2}+\left(\sigma_{3}-\sigma_{1}\right)^{2}}{6}
$$

and $k>0$ is the yield stress in shear (Von Mises criterion for yielding). The range of the possible superseismic solutions for an arbitrary load intensity and arbitrary $\nu$ can be treated only numerically [1].

A simplification arises from the observation that in practical HE applications

$$
P / k \gg 1 \text {. }
$$

Bleich shows that, as $P / k$ increases, the bulk of the energy dissipation due to plastic deformation tends to occur in a very narrow wedge area. This area is asymptotically close to a critical angle $\bar{\alpha}$ for which the basic differential equations of the elastic-plastic material present a singularity. In Von Mises materials, the critical angle is

$$
\tan \bar{\alpha}=\sqrt{D^{2} / \bar{c}^{2}-1},
$$

$\bar{c}$ being the speed of propagation of plastic waves,

$$
\bar{c}=\sqrt{\frac{2(1+\nu)}{3(1-2 \nu)} \frac{\mu}{\rho}} .
$$


Bleich suggests that an approximate solution can be obtained by lumping all the plastic effects in a "shock front" and treating the material elsewhere as entirely elastic. This model is developed here for $\nu>1 / 8$. Behind the $s$ front the yield condition could be violated, and $e_{Y}=\max \left(\sqrt{J_{2}^{\prime}} / k-1,0\right)$ indicates the corresponding error. After the stress tensor is computed, it is necessary to verify that the magnitude of $e_{Y}$ is acceptable.

As displayed in Fig. 1, we incorporate in our model a third discontinuity at $\bar{\alpha}$, consisting of a change (or "jump") of the three principal stresses by the same amount $\overline{\Delta \sigma}$ and by an appropriate change in normal velocity.

The wedge region between the $p$ front and the plastic shock is in a uniform state at the yield point with principal stresses

$$
\frac{\sigma_{1}}{k}=\frac{1-\nu}{\nu} \frac{\sigma_{2,3}}{k}=-\sqrt{3} \frac{1-\nu}{1-2 \nu} .
$$

By imposing the continuity of normal and tangential stresses at the $s$ front, we find the jump at the plastic front

$$
\frac{\overline{\Delta \sigma}}{k}=-\frac{P}{k}+\frac{\sqrt{3}}{2}\left(\frac{1}{1-2 \nu}+\frac{\cos 2\left(\alpha_{P}-\alpha_{S}\right)}{\cos 2 \alpha_{S}}\right)
$$

and the principal stresses after the shear front

$$
\begin{gathered}
\frac{\sigma_{1}}{k}=-\frac{P}{k} \quad \frac{\sigma_{3}}{k}=-\sqrt{3} \frac{\nu}{1-2 \nu}+\frac{\overline{\Delta \sigma}}{k}, \\
\frac{\sigma_{2}}{k}=-\frac{P}{k}+\sqrt{3} \frac{\cos 2\left(\alpha_{P}-\alpha_{S}\right)}{\cos 2 \alpha_{S}} .
\end{gathered}
$$

The deflection $\delta$ can be computed by following the steps indicated in [4] for the linear elastic case. The deformation due to the $p$ and $s$ fronts is of order $\mathcal{O}(k / P)$ with respect to the term due to the plastic shock. If the condition of Eq. (8) holds, the overall deflection is approximated by a hydrostatic compression $P$ at the critical angle $\bar{\alpha}$

$$
\begin{gathered}
u=D(1-2 \nu) \frac{P}{E} \sin \bar{\alpha}^{2}, \\
v=D(1-2 \nu) \frac{P}{E} \sin \bar{\alpha} \cos \bar{\alpha} .
\end{gathered}
$$

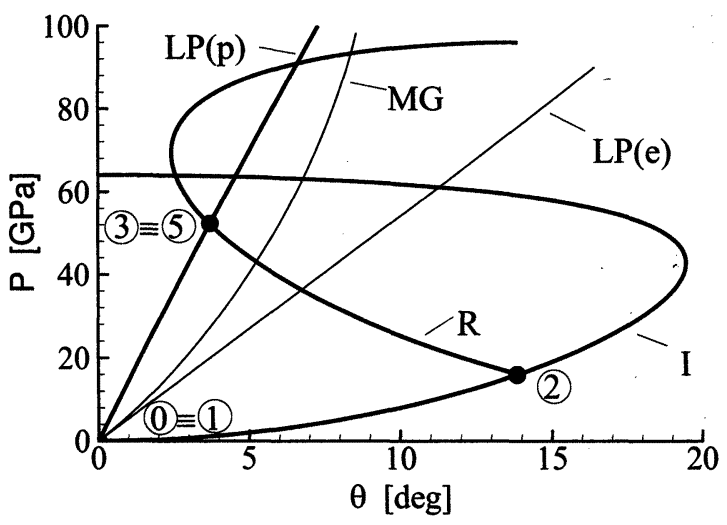

FIGURE 2. Shock polars for Regular Reflection. The labels are as in Fig. 1, but without region 4 and Mach stem

The deflection $\delta$ is given by

$$
\tan \delta=\frac{v}{D+u}
$$

with $u$ and $v$ obtained from Eq. (6) or Eq. (14).

Since $\delta$ has to be equated to the flow deflection $\theta$ at the wave intersection (or node), $\delta(P)$ can be plotted in a pressure versus $\theta$ diagram. The curve will be referred to as the load polar for elastic deformations, LP(e) from Eq. (6), and for plastic deformations, LP(p) from Eq. (14).

\section{FLUID-SOLID INTERACTION}

Shock polars are derived from shock jump relations for an inviscid fluid. This standard procedure is described in [2]. The shock polar diagram for an incident angle $\beta_{2}=30^{\circ}$ (Regular Reflection, or RR) is shown in Fig. 2. It is computed with a perfect gas $\operatorname{EoS}(\gamma=3)$ mimicking a high explosive with density at rest $\rho_{1}=210^{3} \mathrm{~kg} / \mathrm{m}^{3}$ at a pressure $P_{1}=10^{5} \mathrm{~Pa}$.

The confining material is copper, $\nu=0.33$, Young modulus $E=110 \cdot 10^{9} \mathrm{~Pa}$ and density at rest $\rho_{0}=8930 \mathrm{~kg} / \mathrm{m}^{3}$. These parameters correspond to a strong confinement, exhibiting a nontrivial intersection with the incident shock polar I. The weak confinement case, where the load polar is below I and connected to it by an expansion fan, offers less variety of wave patterns and it is not studied here.

Also, we find $e_{Y}<4 \cdot 10^{-4}$ for the range of superseismic speeds $D$ of interest (in Fig. 2, $D=8 \cdot 10^{3} \mathrm{~m} / \mathrm{s}$ ). 
In Fig. 2 the elastic load polar is traced only as a reference, since the ratio $P / k \approx 100$. For comparison, the shock polar of "fluid" copper is also traced (MG). It is calculated by using the Mie-Grüneisen EoS and a linear shock velocityparticle speed relation, $U_{s}=c_{0}+s u_{p}$, with $c_{0}=3940 \mathrm{~m} / \mathrm{s}$ and $s=1.489$. The curve lies between the elastic and the plastic load polars. It should be noted that a rigid interface would generate a Mach Reflection (MR) instead of a RR.

We now briefly consider the more complex case of a MR (not displayed here). It exhibits a triple point in the fluid (TP) and two contact discontinuities (Fig. 1). The problem allows a pseudo-steady solution in the reference moving with TP at an angle $\chi$ with respect to the undeformed interface ahead. In the rigid boundary case, $\chi$ can be found by ignoring the variation of pressure (and, therefore, the effect of curvature) along the stem (straight Mach stem angle [2]), $\alpha=\pi / 2$. This approximation can be extended to a deformable wall, with $\alpha$ now a function of the solid deflection $\delta$ (and, therefore, a function of $P_{4}$ through Eq. (15)).

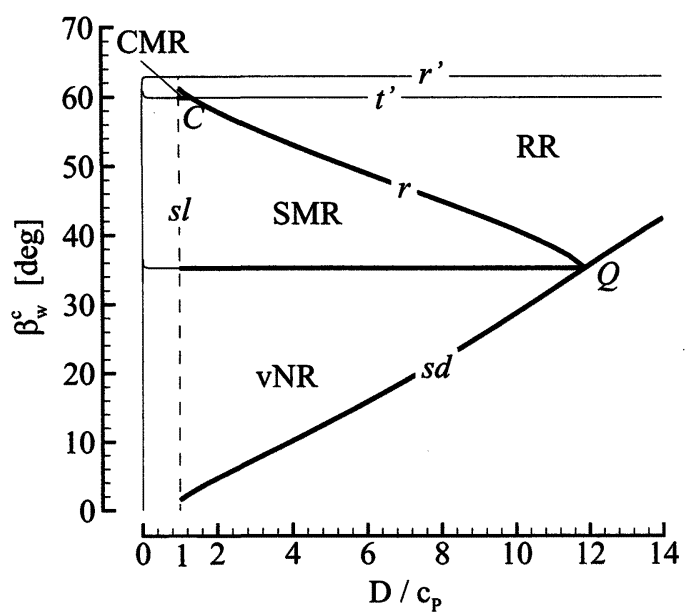

FIGURE 3. Transition boundaries for perfect gas $\gamma=3$ and copper.

To conclude, we consider the modification of a standard transition diagram [2] for a perfect gas (Fig. 3). The complementary wedge angle $\beta_{w}^{c}=90^{\circ}-\beta_{2}$ is plotted versus $D / c_{P}$ in the plastic deformation case. The parameters for the two materials were previously listed. No transition from RR to Double Mach Reflection (DMR) could be computed for a perfect gas with $\gamma>2.28$.

Since the superseismic model applies when $D>c_{P}$, a pseudo-steady solution with a deformable interface can be found only on the right of the superseismic limit (broken line $s l$ ). Transition boundaries in this domain are traced with a thicker line.

In the rigid interface case, the transition between RR and MR would be essentially a straight line, with a limit angle at $\beta_{w}^{c}=62.9^{\circ}$ (line $r^{\prime}$ ). Due to the deformation of the interface, the transition line $(r)$ is appreciably moved downward.

The shock deflection boundary (line $s d$ ) corresponds to the trivial solution $\beta_{w}^{c}=\pi / 2$ in the rigid case. Along $s d$, the wave system degenerates to a single shock, with flow deflection equal to $\delta$. This particular solution was examined in [4] for the elastic case.

The $s d$ line intersects the RR boundary and the boundary between Single Mach Reflection (SMR) and von Neumann Reflection (vNR) at a unique point, $Q$, with no reflected shock and sonic deflected flow.

Finally, the line $t$ separating SMR from Complex Mach Reflection (CMR) intersects the boundary RR-SMR at point $C$. We verified that for higher values of $D / c_{P}$ (line $t^{\prime}$ ), the triple point angle is negative, a condition that identifies non pseudo-steady Irregular Reflections [2].

\section{ACKNOWLEDGEMENTS}

This work was carried out at Caltech ASCI ASAP and funded by Contract B341492 under DOE Contract W-7405-ENG-48.

\section{REFERENCES}

1. Bleich, H., and Nelson, I., J. of Appl. Mech. 3, 819-852 (1966)

2. Ben-Dor, G., Shock Wave Reflection Phenomena, Springer-Verlag, 1992

3. Sternberg, H., and Piacesi, D., Phys. of Fluids 9, $1307-1315$ (1966)

4 Arienti, M., Hung, P., Morano, E., and Shepherd, J.E., to be submitted to J. of Comp. Physics 